\title{
Scanwave: A New Approach to Enhancing Spectral Data on a Tandem Quadrupole Mass Spectrometer
}

\author{
Daniel J. Kenny, Kenneth R. Worthington, and John B. Hoyes \\ Waters Corporation, MS Technologies Centre, Manchester, United Kingdom
}

\begin{abstract}
A new type of mass analyzer is described, which allows low-resolution axial ion ejection to be obtained from a traveling wave based, stacked ring collision cell. Linking this ejection temporally with the scanning of the second quadrupole of a tandem quadrupole mass spectrometer provides an improvement in sampling duty cycle, which results in significant signal intensity improvements for scanning acquisitions such as product ion spectra. A near $100 \%$ storage efficiency is enabled by a split cell design, which allows ion fragmentation and accumulation to be performed in one section of the collision cell whilst previously accumulated ions are simultaneously ejected from the rear of the cell. These characteristics combine to give an $\mathrm{m} / \mathrm{z}$-dependent signal gain of 7-20× over a conventional scanning quadrupole for a 1000 Th scan. The ability to swap very rapidly from a non-enhanced mode of operation to an enhanced mode whilst retaining the existing sensitivity, speed, and functionality of a conventional tandem quadrupole mass spectrometer is also described. (J Am Soc Mass Spectrom 2010, 21, 1061-1069) (C) 2010 American Society for Mass Spectrometry
\end{abstract}

$\mathrm{Q}$ uadrupole mass filters (QMF) are common analytical devices used in a wide variety of mass spectrometry equipment [1]. However, a major drawback of these devices, when they are operated in a scanning mode to generate a mass spectrum, is low sensitivity due to poor sampling duty cycle. For example, if a QMF scans an $m / z$ range of $x$ with the quadrupole resolving to give peaks of width $y$ at half height, then the duty cycle of the quadrupole mass filter will be $y / x$. Conventional QMFs used in tandem quadrupole instruments may typically have a duty cycle as low as $0.1 \%$.

Various modifications to the QMF have led to the creation of several different linear ion traps (LIT) [2]. LITs generally have an increased space charge capacity compared with 3D/Paul ion traps [3] and have been successfully employed in many application areas. LITs have also been incorporated into several hybrid geometry mass spectrometers [4-7]. These devices either eject ions radially [6, 7] or axially [8, 9]. One recent approach to improving the quality of spectral data on a tandem quadrupole platform has seen the second resolving quadrupole replaced with a linear ion trap to form a hybrid instrument [2].

A different approach to improving spectral data on a tandem quadrupole is to retain the second resolving quadrupole but to operate it as the second mass ana-

Address reprint requests to Dr. D. J. Kenny, Waters Corporation, MS Technologies Centre, Floats Road, Wythenshawe, Manchester M23 9LZ, UK. E-mail: daniel_kenny@waters.com lyzer in a "linked-scan" configuration [8-10]. The linked scan approach uses two mass analyzers operating in synchronism; the first, a low-resolution device, is used to deliver ions to the second, higher resolution device such that ions arrive just as they are to be analyzed rather than in a continuous stream. In this way, the sampling duty cycle may be improved, which leads to an improvement in signal intensity and, hence, instrument sensitivity. This concept has previously been used to increase the duty cycle of an orthogonal acceleration time of flight (oa-TOF) mass spectrometer by coupling it with both an axial TOF [11] and an ion mobility separator (IMS) [12] acting as the low-resolution device.

The concept of a linked scan as it applies to a scanning QMF can be understood by considering the two hypothetical situations portrayed in Figure 1. Represented here is a plot of the ion current incident at the entrance of a QMF (dotted lines) and the ion current at its exit (solid lines) for ions of three different $\mathrm{m} / \mathrm{z}$ over the course of a single scan lasting for a time period $t$. Each of the three ions has a different ion current and the total number of ions for each is correspondingly represented by the shaded areas A, B, and C. For a perfect device, where there are no transmission losses, the height of the mass spectral peaks detected at the exit of the QMF as a result of scanning the quadrupole is the same as the constant incoming ion current in each case and, hence, constitutes the normal sensitivity of a conventional scanning QMF. Figure $1 b$ represents a linked scan experiment where the same perfect QMF is 

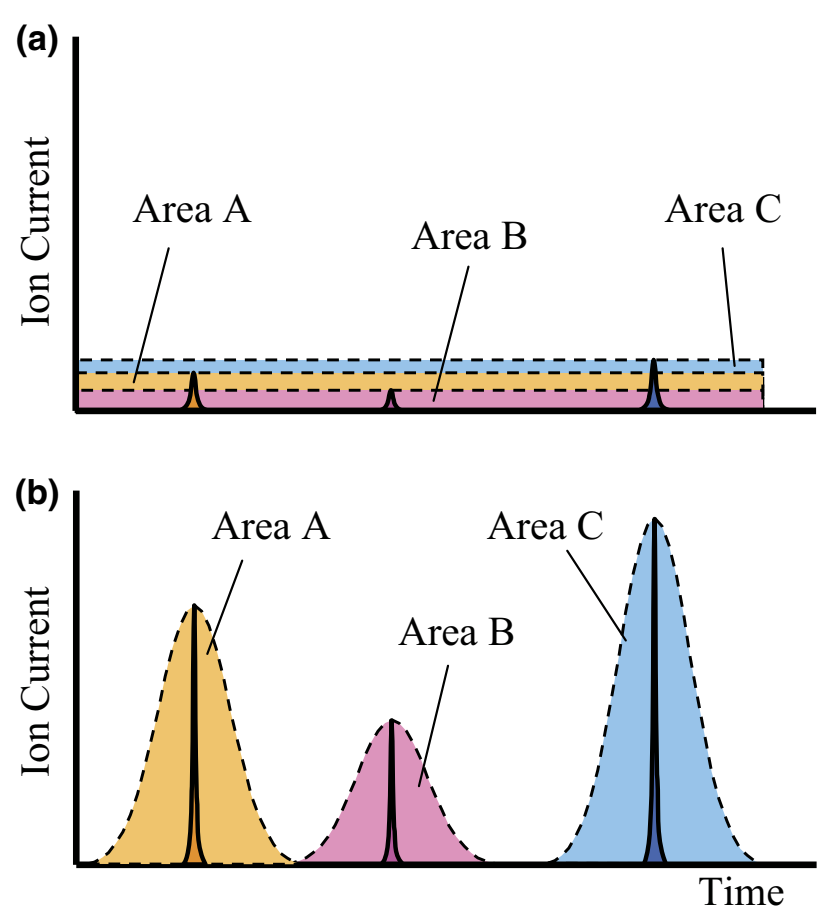

Figure 1. (a) The ion currents incident at the entrance of a QMF (dotted lines) and the resultant mass spectral peaks (solid lines) for three ions of different $\mathrm{m} / \mathrm{z}$ in a conventional mass spectrometer. (b) The ion currents and mass spectral peaks in a linked scan experiment.

preceded by a device that has accumulated all of the incident ions and has then been used to eject them to the QMF in synchrony. In this idealized experiment, the number of ions and, hence, the areas A, B, and C incident upon the entrance of the QMF are the same as in the previous experiment, but the peak ion current is now increased due to the concentrating effect of ion storage and subsequent mass separation. Consequently the height of the mass spectral peak detected at the exit of the QMF is also substantially higher than in the previous experiment, thus realizing an improvement in sensitivity. It should be emphasized that this linked scan methodology cannot be used to increase duty cycle on a standard tandem quadrupole; the key is to store the ions in a device that can mass-selectively eject them.

In this paper, we present a novel mass analyzer, Scanwave, which realizes a sensitivity improvement when operated in a linked scan manner compared with a conventional scanning QMF. This mass analyzer is based upon a stacked ring ion guide geometry and is located at the rear of the collision cell in a tandem quadrupole instrument. A novel rf phase switching method allows the collision cell to function in two modes of operation. In one mode, it acts as a conventional collision cell whereas in the second mode, the front of the cell is used to fragment and accumulate ions whilst the rear of the cell mass selectively delivers ions to the second resolving quadrupole.

\section{Experimental}

The experiments presented here were performed on a prototype Xevo TQ MS instrument (Waters Corporation, Manchester, UK). This instrument configuration is identical to that of a standard tandem quadrupole with the inclusion of the Scanwave collision cell between Q1 and Q2 in place of a conventional collision cell. In this context, we define a conventional collision cell as one that acts purely as a fragmentation and transmission device, i.e., a multipole device such as a hexapole or else a stacked ring device or curved ion guide, rather than the stricter definition of a straight, non-resolving quadrupole. The construction of the Scanwave cell is very similar to that used previously for the production of traveling wave ion guides (TWIGs) in that a total of 122 ring electrodes are mounted between two printed circuit boards (PCBs), which are used to provide the electrical connections to the electrodes. The Scanwave device is segmented to allow it to be electrically divided into discrete sections, each of which is controlled using an individual TWIG electronics unit allowing independent control of rf amplitude. The rf frequency and phase may be locked to a common reference clock permitting seamless operation as a single cell. Alternatively, this arrangement allows control of relative phase difference between the sections. Argon was used as the collision gas for all experiments and was maintained at a pressure of $\sim 3.5 \mathrm{E}-3$ mbar. The Scanwave collision cell ejects ions in reverse $\mathrm{m} / \mathrm{z}$ order and consequently the quadrupoles were programmed to scan from high to low $\mathrm{m} / \mathrm{z}$ rather than the normal method of scanning from low to high.

\section{Stacked Ring Ion Guides and Scanwave}

The Scanwave device is based upon TWIG devices, which have been described previously $[13,14]$ and so will only be described briefly here. The TWIG comprises a series of planar ring electrodes arranged orthogonally to the ion transmission axis where opposite phases of an rf voltage are applied to adjacent electrodes. To propel ions through the TWIG a transient DC voltage is superimposed on the rf applied to a pair of adjacent electrodes in a repeating sequence along the length of the device. The series of potential hills so generated are subsequently applied to the adjacent pair of electrodes downstream at regular time intervals providing a continuous sequence of traveling waves. The ions within the device are driven away from the potential hills and consequently are carried through the device with the waves, minimising their transit time.

Close inspection of the flat central region of the TWIG pseudopotential reveals the existence of small axial corrugations highlighted in Figure 2. The maximum height of these corrugations along the central axis is $\sim 130 \mathrm{mV}$ for a singly charged ion of $\mathrm{m} / \mathrm{z} 100$ in a device using $5 \mathrm{~mm}$ diameter apertures, a center-tocenter spacing of $1.5 \mathrm{~mm}$, and an rf voltage of 2.65 


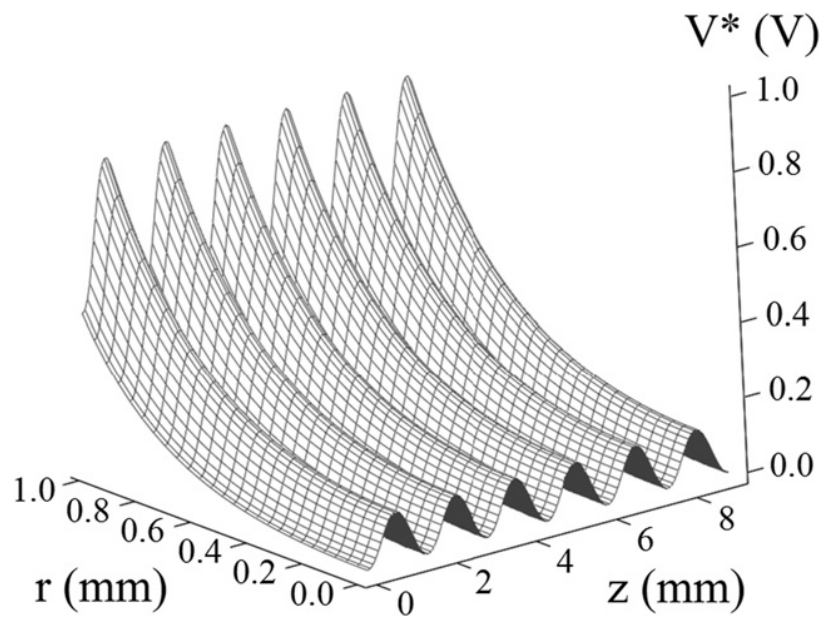

Figure 2. The radial pseudopotential for a TWIG highlighting the existence of small axial corrugations.

$\mathrm{MHz} / 400 \mathrm{~V}_{p-p}$. The height of the traveling wave that is normally applied to the TWIG is of the order of $1-5 \mathrm{~V}$ and so these small, normally undesirable, axial corrugations do not impede the progress of ions within the device. However, the concept behind the Scanwave device is to purposefully increase the height of one or more of these axial corrugations to the extent that they provide a barrier, which will actually prevent the axial transit of ions, unless a traveling wave of sufficient magnitude acts to propel the ions past the barrier.

There are numerous possible approaches to increasing the height of the axial corrugations in a TWIG, for example geometrical changes such as increasing the spacing between rings or decreasing their internal diameter, or alternatively increasing the rf voltage, decreasing the rf, or altering the phase of the rf applied to adjacent plates. The chosen implementation is shown in Figure 3a, which schematically represents a cross section of a region of the collision cell and where the ' + ' and ' - ' symbols reflect the phase of the rf applied to each lens. One pair of rings (hatched) are driven using an independent rf power supply, which is phase-locked to the rf supply powering the adjacent rings, thus allowing, in one mode of operation, the Scanwave device to be ion optically identical to a standard TWIG. However, as shown in Figure 3b, when mass separation is required, the phase of the rf applied to a single pair of rings is shifted by $180^{\circ}$. This action effectively creates two "thicker" rings with a correspondingly greater center-to-center separation, the result of which is to increase the height of the axial corrugation at this point, thus forming a sufficiently tall barrier to prevent ions from being transported onward. For the rf conditions discussed above, the corrugation height for an ion of $\mathrm{m} / \mathrm{z} 100$ increases to $\sim 4.7 \mathrm{~V}$, which is sufficient to impede the transport of ions past the barrier and act as the basis of an analytical separation.

It should be noted that the linked scan approach is only suitable for acquisitions where the second resolv- ing quadrupole is scanned. For acquisition types where this quadrupole is held static, there is no benefit to this approach and so it is necessary to operate the collision cell in a conventional manner, i.e., as a fragmentation and transmission device, not as an accumulation and ejection device. The advantage of the phase switching approach is that it allows the device to be operated as a conventional TWIG collision cell, thus allowing all standard modes of operation of a tandem quadrupole to be performed on the instrument, e.g., multiple reaction monitoring (MRM), without degradation in sensitivity, speed, or other performance characteristic. Alternatively, when desired, the collision cell may be switched rapidly into the linked scan mode of operation for acquiring enhanced spectral data. Forming a pseudopotential barrier using a purely mechanical approach would not allow this dual mode operation and would potentially degrade the standard operation of the instrument.

\section{Results}

\section{Mass Separation Proof of Principle}

To determine what degree of mass separation could be achieved by forming an axial barrier in the collision cell
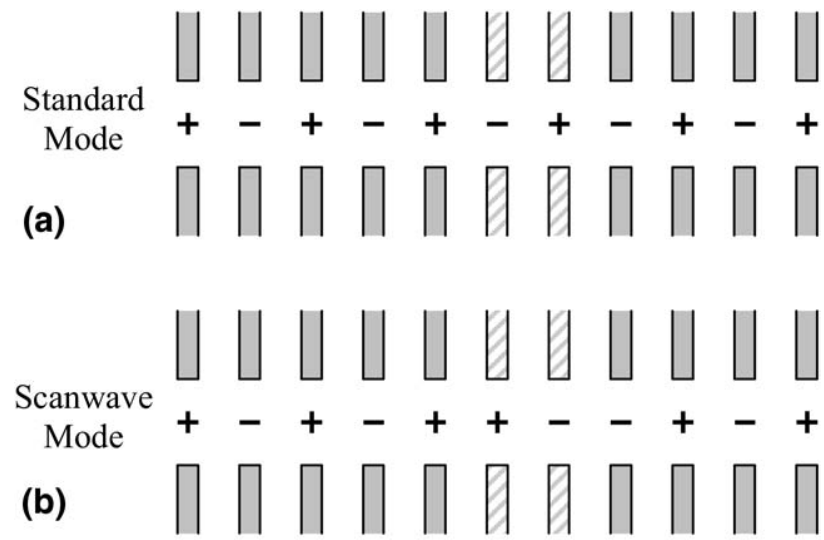

(c)

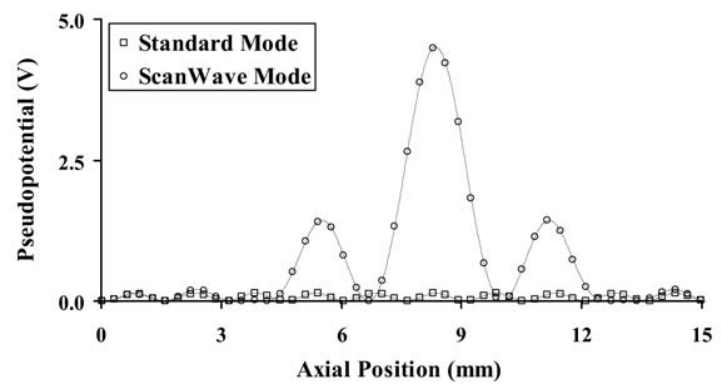

Figure 3. Schematic representation of the rf phases applied to the Scanwave device. (a) Standard T-Wave mode used for MRM and other non-enhanced acquisition modes. (b) Scanwave mode where the phase of the rf applied to a single pair of electrodes (hatched) has been reversed. (c) Plot of the axial pseudopotential along the center of the collision cell in both modes of operation. 
by the phase swapping method described above, various ejection methods were tested. The method that provides the best mass separation is when a traveling wave with a fixed pulse height and velocity is used to continuously impel ions towards the rf barrier, the height of which is steadily reduced by decreasing the confining rf voltage applied to the electrodes. At the start of an experiment the height of the rf barrier is set to its maximum and consequently all ions that do not have sufficient kinetic energy to traverse the barrier are confined. As the experiment progresses the $\mathrm{rf}$ barrier is decreased, allowing ions of a progressively lower $m / z$ to traverse past the barrier as their kinetic energy, imparted by the traveling wave, exceed the barrier potential. In this manner, the device behaves as a high pass filter where the edge of the filter is scanned down from high to low $m / z$, depleting the device of the accumulated ions as it does so. For these proof-of-principle experiments, mass separation data were obtained by accumulating ions within the source hexapole by applying a DC potential to the differential pumping aperture between the source ion guide and Q1, before periodically releasing them into the Scanwave cell. Applying a DC offset between Q1 and the Scanwave cell allowed CID to be performed on the precursor ion isolated by Q1. The resultant fragment ions were ejected over a period of $200 \mathrm{~ms}$ with Q2 set to transmit a single $\mathrm{m} / \mathrm{z}$. This allowed chromatograms of the temporal evolution of the ion current for several various $m / z$ to be recorded. Typical chromatograms are presented in Figure $4 \mathrm{a}$ for fragment ions of Glu-fibrinopeptide. It is immediately apparent that, as expected, ions are ejected in reverse $m / z$ order, i.e., ions of higher $m / z$ are ejected first, and that the peak widths are quite broad, implying only modest mass resolution.

To characterize the ion ejection, data were acquired to determine the separation behavior of the Scanwave device as a function of TW pulse height, TW pulse velocity, and separation timescale. By way of illustration, the separation plots obtained for different TW pulse heights with a TW velocity of $140 \mathrm{~m} / \mathrm{s}$ and an experiment time of $200 \mathrm{~ms}$ are shown in Figure $4 \mathrm{~b}$. The separation is nonlinear and a larger pulse height leads to ions being ejected over a taller rf barrier and, hence, at an earlier time. This behavior is consistent with the premise that ejection occurs when the energy imparted to the ion by the traveling wave is sufficient for the ion to overcome the rf barrier as a greater TW pulse height will result in higher ion energy. Variation of $\mathrm{m} / \mathrm{z}$ ejection characteristic with pulse height is useful as it provides a way of controlling the $m / z$ range that may be ejected, a key requirement for any separation device. As shown, for small TW pulse heights of $<200 \mathrm{mV}$, it is possible to obtain mass separation beyond $m / z$ 2000, allowing large molecules such as peptides to be separated. Further investigation has shown that by using a nonlinear rf ramp, linear mass separation may be achieved, but these specific details are outside the scope of the current article.

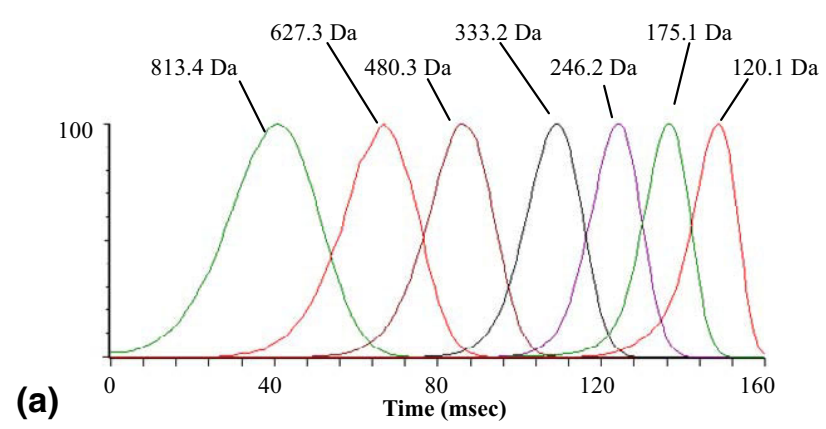

(b)

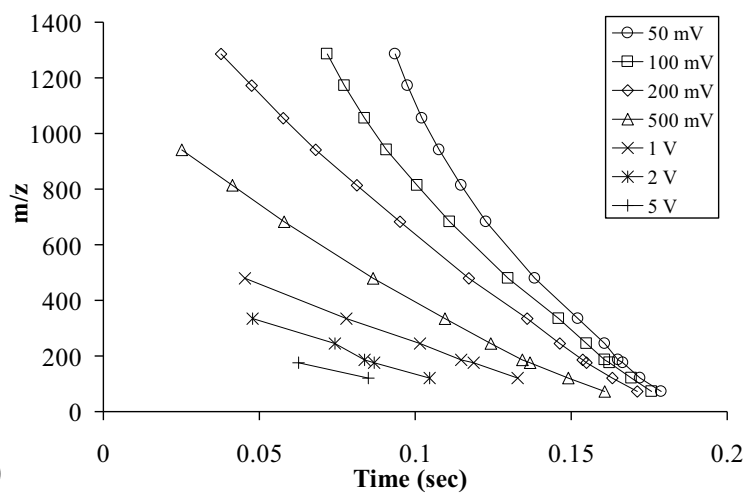

(c)

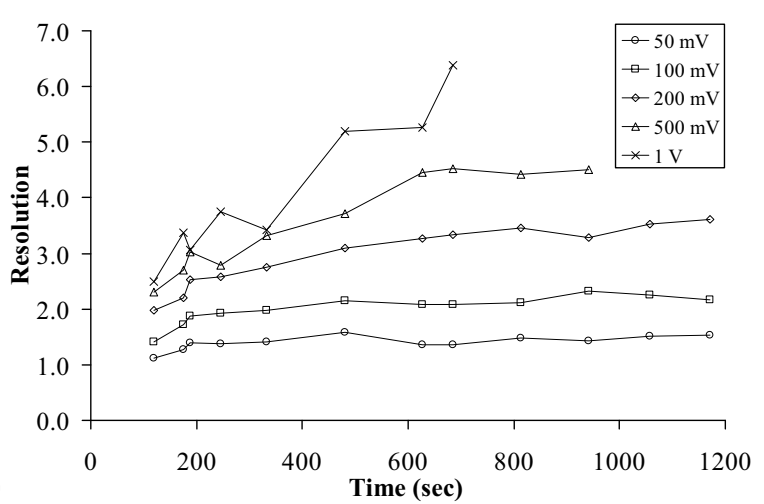

Figure 4. (a) Typical mass chromatograms obtained for fragment ions of Glu-fibrinopeptide by ejecting ions out of the Scanwave device, whilst setting Q2 to resolve at the appropriate $m / z$ and measuring the resultant ion current received at the detector. (b) Plots of the separation behavior of the Scanwave device when the rf amplitude is ramped linearly with time and the TW pulse height is kept constant. (c) Typical mass resolutions obtained when using a non-linear rf ramp to obtain linear mass separation.

The mass resolution of the Scanwave device was determined for five different traveling waves amplitudes as highlighted in Figure 4c, which compares the measured mass resolutions obtained as a function of $m / z$. The data shows a clear increase in resolution with increasing $\mathrm{m} / \mathrm{z}$. Furthermore, a larger traveling wave pulse height leads to greater resolution, albeit at the expense of a reduced $m / z$ range. Clearly, the measured mass resolutions, which vary between 1.0 and 6.5, are not sufficient in terms of utilizing this device as a stand alone mass analyzer. However, even 
this modest mass resolution can be utilized to greatly increase the sensitivity of a scanning quadrupole mass filter.

Another variable that has been investigated is the effect of gas pressure within the device upon the separation behavior and resolution. It was found (data not shown) that there is no significant effect at normal collision cell pressures (1E-3 to 1E-2 mbar) although below 1E-3 mbar the increased mean free path and the consequently lower thermalization effect of the gas leads to a proportion of ions that are not sufficiently cooled escaping out of the collision cell without being separated.

\section{Space Charge Effects}

There is a finite charge capacity for any ion storage device beyond which its performance begins to degrade and where ultimately the device cannot hold any further charges. Overfilling an analytical 2D/3D ion trap leads to loss of mass resolution and of mass accuracy, a result of the perfect quadrupolar electric field becoming distorted by the presence of the large number of charges being focussed into close proximity [15]. A common method used to avoid overfilling ion traps such as LITs has been to perform a preliminary scan to determine the intensity of the incoming ion current and to then fill the trap for a calculated period of time so that a specific number of ions are accumulated [16, 17]. This method is generally referred to as either automatic gain control (AGC) or dynamic fill time (DFT). For intense ion currents such as those from liquid chromatography, the fill time can be very short, resulting in a low sampling duty cycle that consequently results in reduced sensitivity. It was therefore a major objective for the Scanwave device to have a sufficiently high space charge capacity to cope with typically encountered ion currents and so avoid the necessity of dynamically controlling the fill time and, hence, the loss of sensitivity and increase in experiment time associated with this approach.

To ascertain the effect of space charge on the performance of the Scanwave device, mass chromatograms, as already described and presented in Figure $4 a$, were acquired for the $246 \mathrm{Da}$ fragment of Glufibrinopeptide, where the total number of ions accumulated in the storage region of the Scanwave device was varied from 400 ions to 400,000 ions. The number of ions was controlled by altering the ion current (as measured with both quadrupoles set to transmit the precursor and with no trapping in the Scanwave cell) such that a fixed fill time resulted in the requisite number of ions being accumulated. The vertical dotted line overlay of the chromatograms in Figure 5 plainly intersects the peak of the three lower ion count chromatograms, indicating that the separation behavior is essentially unchanged between 400 and 40,000 ions. Close inspection reveals that there is a small shift to earlier time/higher rf voltage for the

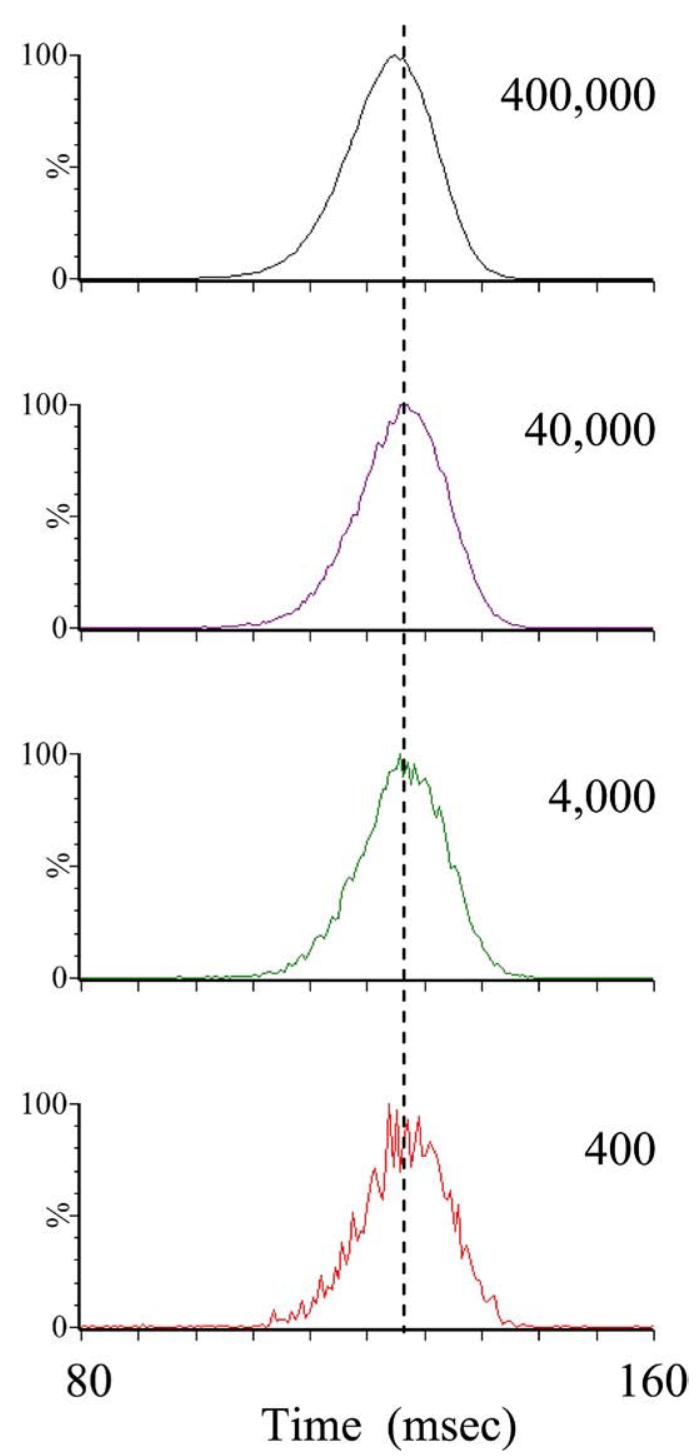

Figure 5. Effect of space charge on the ejection of the $m / z 246$ Da fragment ion of Glu-fibrinopeptide from the Scanwave device. The number of ions indicated relate to the total number of ions accumulated prior to separation of the fragments.

400,000 ion case, most likely due to the large concentration of charge reducing the effective height of the rf barrier and, hence, the ions are ejected at higher applied rf amplitude. However, in all four chromatograms, the peak width is essentially unaffected by increasing the number of charges.

It should be noted that the effect upon the mass spectral data acquired in a linked scan experiment of a space charge induced shift to earlier ejection time is a small decrease in sensitivity as the top of the peak "elutes" from the Scanwave device before the quadrupole transmitting that particular $m / z$. This small sensitivity reduction is in contrast to the behavior of a conventional linear ion trap where increased space charge leads to both mass resolution and mass accuracy degradation, thus highlighting the robustness of this low-resolution device. 


\section{Split Cell Design}

A consequence of the inherent structure and design of the TWIG is that it readily lends itself to segmentation into separate regions. In the Scanwave device, full use of this ability has been used to split the single collision cell into two distinct regions, with the aim of utilizing the front region to fragment and accumulate ions whilst previously accumulated ions are being ejected from the rear of the device. The motivation for this design is to allow ion accumulation after the first mass analyzer (Q1) so as to minimize the quantity of charge being accumulated [5].

In the linked scan mode, where the collision cell is segmented, the front ion accumulation section must also act as the collision cell. It must therefore be of a sufficient length to ensure comparable fragmentation and efficiency compared with when the Scanwave collision cell is used as a conventional collision cell, without having to alter the gas pressure or the collision energy. Conversely, the ejection region should not be too short or else its space charge capacity would be unduly affected. The chosen design consists of a storage region, which occupies the first two-thirds of a standard TWIG collision cell and an ejection region that occupies the remaining third, as this was found to satisfactorily fulfil both design criteria. Two independent TWIG electronics units are used to control these two sections, thus allowing independent control of rf voltage, TW height, and TW velocity when the mass spectrometer is being operated in a linked scan mode. Furthermore, a third TWIG controller is used to control the rf barrier electrodes as discussed previously. For those experiments where Scanwave functionality is not employed (for example MRM), it is necessary for the Scanwave device to act as a conventional TWIG collision cell and so the three TWIG controllers are phase locked, both in terms of the confining $\mathrm{rf}$ that is applied to the electrodes, as well as the traveling waves that traverse the length of the cell. Effectively, this allows the Scanwave device to be ion optically identical to a standard TWIG device, but with the ability to rapidly swap to and from Scanwave linked scan mode.

Furthermore, an advantageous aspect of storing ions in the collision cell is that it allows ion accumulation to be performed after the first mass selection stage. For MS/MS acquisitions, this means that only the isolated precursor and its fragment ions are accumulated and not unwanted ions such as those arising from solvent, matrix, or co-eluting compounds. For MS acquisitions, there is a small benefit in accumulating after the first analytical quadrupole as the low mass cutoff may be used to eliminate unwanted low $\mathrm{m} / \mathrm{z}$ ions such as those arising from solvent.

A typical storage and ejection cycle is depicted in the series of illustrations in Figure 6. Initially, the DC barrier at the end of the storage region is set high to allow ions to accumulate. During the interscan period between two scans the barrier is lowered and a traveling wave used to rapidly and efficiently transport ions from the storage region to the ejection region. At the start of the subsequent scan, the DC barrier is raised so that ions may again be accumulated whilst ions are ejected out of the ejection region. It should be noted that normally any ions incident upon the mass spectrometer during the interscan period are lost. However, with the current scheme it is possible to accumulate ions even during the interscan thus realising a small additional improvement in duty cycle and hence a small improvement in signal intensity that scales with shorter scan times. Once ions have been transferred to the ejection region, the appropriate TW for the $\mathrm{m} / \mathrm{z}$ range of the experiment is started and the rf voltage applied to the barrier is ramped downward, following a nonlinear profile to eject ions in synchrony with the scanning of Q2. Note that because the Scanwave device ejects ions in reverse $\mathrm{m} / \mathrm{z}$ order, it is necessary to scan Q2 in reverse, i.e., from high to low $\mathrm{m} / \mathrm{z}$. This has no effect upon sensitivity, resolution, or mass accuracy compared with scanning forwards.

\section{Data Enhancement}

Because the addition of the linked-scan functionality of the Scanwave device has required no changes to be made to the standard tandem quadrupole geometry, this allows direct comparison of a conventional scanning quadrupole spectrum with a Scanwave linked scan spectrum, i.e., a conventional product ion spectrum can be acquired by using the Scanwave cell as a transmission fragmentation device, and an enhanced spectrum can be acquired by phase swapping the lenses within the cell and performing a linked scan experiment, as already described. Performing a comparison in this manner means that the same mass spectrometer is used to obtain both datasets and so any improvements in sensitivity can be ascribed solely to the new acquisition method and not to other confounding factors such as different instrumentation. Figure 7a shows a conventional scanning quadrupole MS/MS spectrum of 1 $\mathrm{pmol} / \mu \mathrm{L}$ Glu-fibrinopeptide infused at a flow rate of 10 $\mu \mathrm{L} / \mathrm{min}$. The quadrupole was scanned at a rate of 5000 $\mathrm{Da} / \mathrm{s}$ from 100 to $1100 \mathrm{Da}$. The collision cell pressure was set at $\sim 3.5 \mathrm{e}-3$ mbar and a collision energy of $35 \mathrm{~V}$ was applied. Figure $7 \mathrm{~b}$ shows the corresponding Scanwave linked scan spectrum acquired immediately after the conventional spectrum using the same collision cell pressure, collision energy, and quadrupole scan rate. Qualitatively, the spectra are very alike in that they show a similar pattern of fragmentation, except for the obvious increase in signal intensity for the Scanwave data, due to the identical method of fragment ion formation in both modes of operation. However, there is a noticeable decrease in the relative intensity of the ion at $\mathrm{m} / \mathrm{z}$ 187. Possibly, this ion is metastable and is therefore detected in the conventional scan where the 

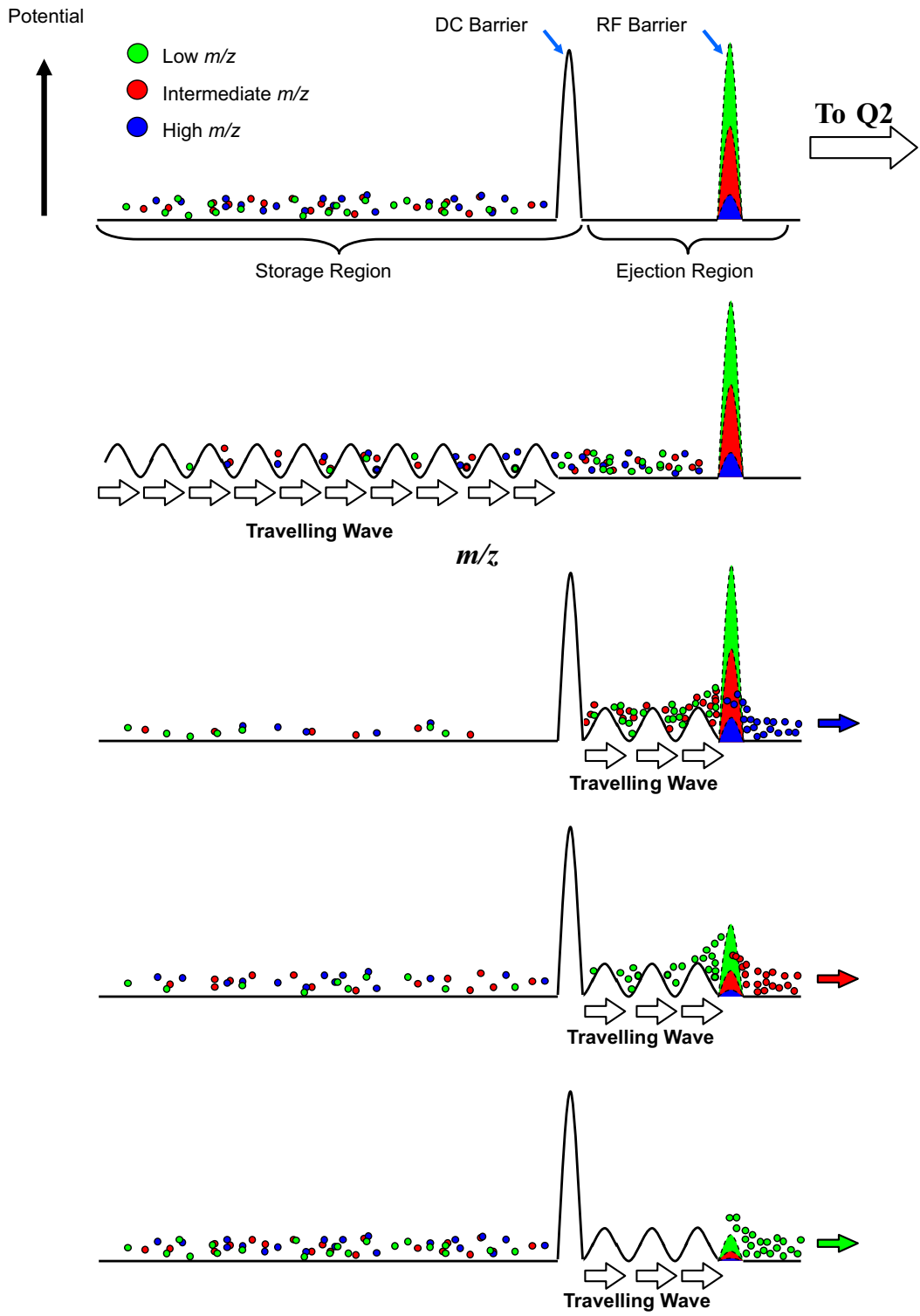

Figure 6. Time-line of a Scanwave linked scan experiment. Ions are initially accumulated before being transferred to the Ejection Region. Whilst these ions are sequentially ejected, simultaneous accumulation of further ions occurs in the Storage Region leading to 100\% sampling efficiency.

timescale between ion creation and ion detection is of the order of $100 \mathrm{~s} \mu \mathrm{s}$, but is not detected in the linkedscan because the ion decomposes during the ion accumulation/ejection cycle, which is potentially several hundred $\mathrm{ms}$, i.e., up to three orders of magnitude difference in timescale. Figure 8 shows the gain in signal intensity obtained as a function of $\mathrm{m} / \mathrm{z}$. Gains of $>20 \times$ can be seen at low $\mathrm{m} / \mathrm{z}$, which slowly decrease with increasing $\mathrm{m} / \mathrm{z}$ to $\sim 7 \times$ at $\mathrm{m} / \mathrm{z} 1000$.

An important feature of the stacked ring geometry of the Scanwave device is its broad $\mathrm{m} / \mathrm{z}$ range. This allows acquisitions that cover a sizeable mass range without significant loss of sensitivity at low $\mathrm{m} / \mathrm{z}$ attributed to the well defined low mass cut off associated with devices of quadrupolar geometry. This avoids previous approaches used with linear ion traps, where multiple spectra are acquired and then stitched together to cover the required mass range [8].

Because the Scanwave device is being used purely as a means of delivering ions to the scanning quadrupole, the mass resolution and mass accuracy of data acquired when performing a linked scan experiment should be identical to that of a conventional scanning quadrupole experiment provided that the Scanwave device is not significantly influencing the mean energy or the energy spread of the ions exiting the collision cell. To test this, data (not shown) were acquired in both modes of operation to compare peak widths and mass positions. No significant variation in mass resolution or mass accuracy between the two experiments was seen when the same mass calibration was applied to both datasets. A detailed account 


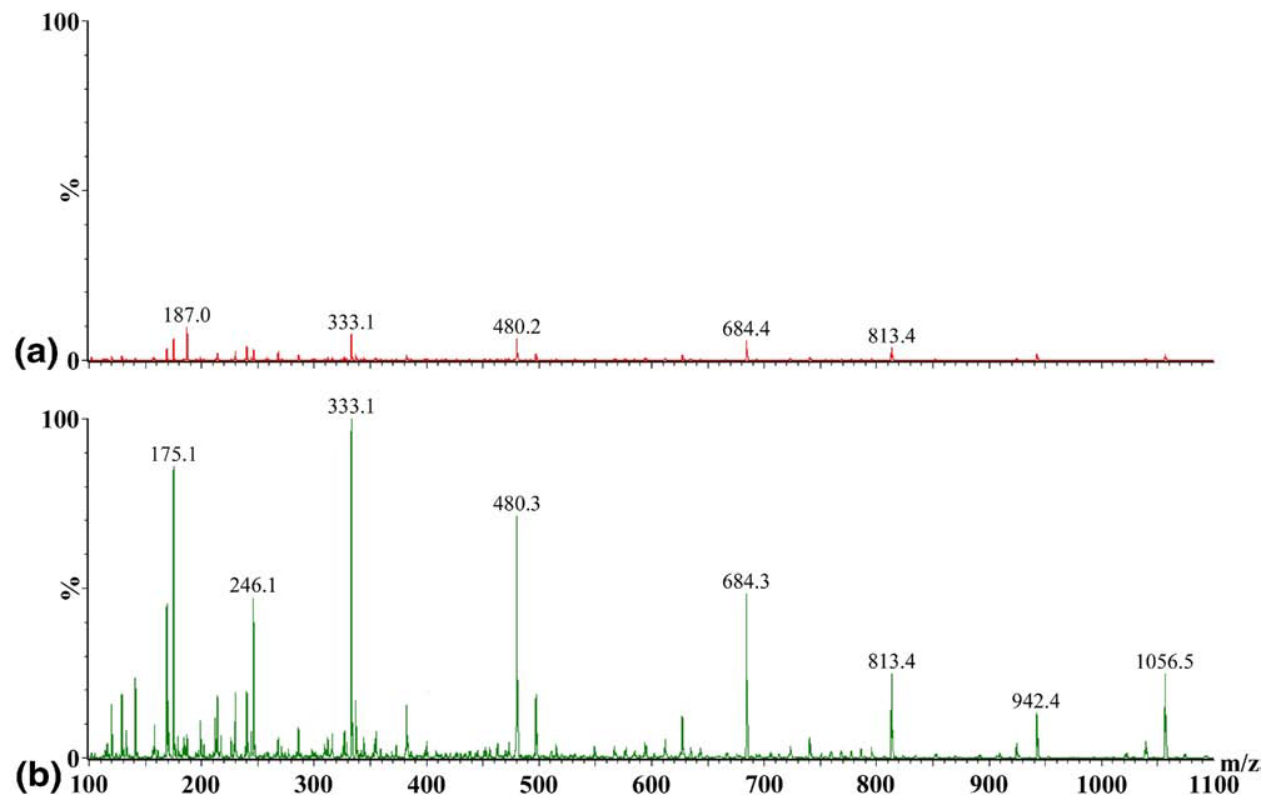

Figure 7. Comparison of scanning quadrupole mass spectra of the fragments of Glufibrinopeptide in (a) conventional operation and (b) Scanwave linked scan operation.

of the experimental performance and benefits of this new device will be published at a later date.

\section{Conclusions}

This work has demonstrated that low-resolution mass separation, when linked with an existing mass analyzer, can lead to significant improvements in signal intensity and, hence, sensitivity for scanning quadrupole acquisitions on a tandem quadrupole platform. A split collision cell design allows the Scanwave device to effectively have a $100 \%$ sampling efficiency by allowing simultaneous ion accumulation and ion ejection. This combination has shown that signal intensity improvements of up to $20 \times$ may be achieved in some situations. Of particular importance is that this additional functionality does not

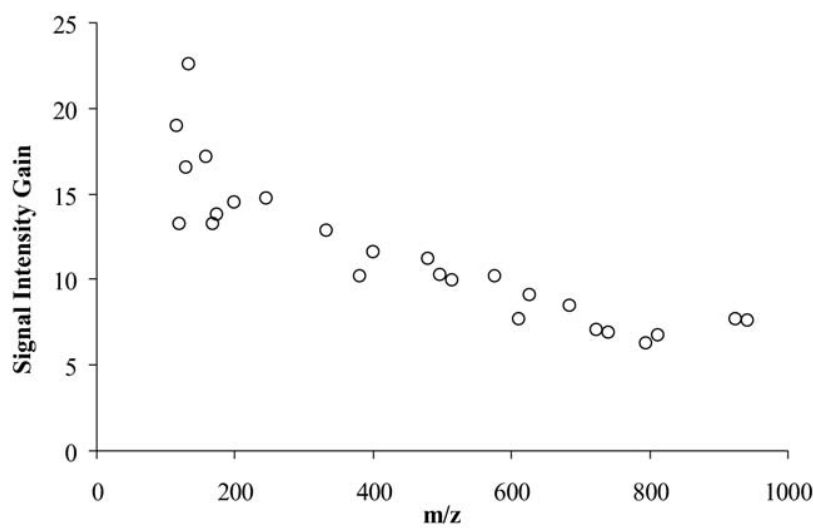

Figure 8. Ratio of signal intensity between Scanwave and conventional experiments as a function of $m / z$ showing gains of $>20 \times$ at low $m / z$, and $>7 \times$ at high $m / z$. compromise the existing sensitivity, speed, or functionality of the tandem quadrupole instrument. This approach further allows rapid switching between enhanced and non-enhanced modes of operation. In addition, the Scanwave device has a very large space charge capacity, which negates the need to limit the number of ions allowed into the device.

\section{Acknowledgments}

There are many people to whom the authors are indebted for their help and assistance throughout the course of this research, particularly Steven Pringle, Jason Wildgoose, and David Gordon. Thank you also to Bob Bateman, Martin Green, and David Little for helpful discussion and comments.

\section{References}

1. Dawson, P. H. Quadrupole Mass Spectrometry and Its Applications. AIP Press: Woodbury, NY, 1995; p 1-349.

2. Douglas, D. J.; Frank, A. J.; Mao D. Linear Ion Traps in Mass Spectrometry. Mass Spectrom. Rev. 2005, 24, 1-29.

3. Campbell, J. M.; Collings, B. A.; Douglas, D. J. A Linear Ion Trap Time-of-Flight System with Tandem Mass Spectrometry Capabilities. Rapid Commun. Mass Spectrom. 1998, 12, 1463-1474.

4. Schwartz, J. C.; Senko, M. W.; Syka, J. E. P. A Two-Dimensional Quadrupole Ion Trap Mass Spectrometer. J. Am. Soc. Mass Spectrom. 2002, 13, 659-69.

5. Makarov, A.; Denisov, E.; Kholomeev, A.; Balschun, W.; Lange, O.; Strupat, K.; Horning, S. Performance Evaluation of a Hybrid Linear Ion Trap/Orbitrap Mass Spectrometer. Anal. Chem. 2006, 78, 2113-2120.

6. Hager, J. W.; Yves Le Blanc, J. C. Product Ion Scanning Using A Q-q-Q Linear Ion Trap (QTrap) Mass Spectrometer. Rapid Commun. Mass Spectrom. 2003, 17, 1056-1064.

7. Hashimoto, Y.; Hasegawa, H.; Satake, H.; Baba, T.; Waki, I. Duty Cycle Enhancement of an Orthogonal Acceleration TOF Mass Spectrometer Using an Axially-Resonant Excitation Linear Ion Trap. J. Am. Soc. Mass Spectrom. 2006, 17, 1669-1674.

8. Hoyes, J. B. Ion Extraction Devices, Mass Spectrometer Devices, and Methods of Selectively Extracting Ions And Performing Mass Spectrometry. US Patent 7405401, 2008. 
9. Hoyes, J. B. A Mass Spectrometer with Improved Duty Cycle. GB Patent Application 20060016878D, 2006.

10. Giles, K.; Green, M.; Pringle, S. D.; Wildgoose, J. L. Method and Apparatus for the Mass-Selective Release of Ions from an Ion Trap. GB Patent Application 20060011062, 2006

11. Pringle, S. D.; Wildgoose, J .L.; Giles, K.; Worthington K.; Bateman R. H. An Investigation into a Method of Improving the Duty Cycle on OA-TOF Mass Analyzers. Proceedings of the 52nd ASMS Conference on Mass Spectrometry; Nashville, TN, 2004.

12. Wildgoose, J. L.; Pringle, S. D.; Giles, K.; Bateman, R. H.; Bajic, S. A Comparison of Methods of Improving the Duty Cycle on Orthogonal TOF Mass Analyzers. Proceedings of the 53rd ASMS Conference on Mass Spectrometry; San Antonio, TX, 2005.
13. Giles, K.; Pringle, S. D.; Worthington, K. R.; Little, D.; Wildgoose, J. L.; Bateman, R. H. Applications of a Traveling Wave-Based Radio-FrequencyOnly Stacked Ring Ion Guide. Rapid Commun. Mass Spectrom. 2004, 18 2401-2414.

14. The Traveling Wave Device Described Here is Similar to That Described by Kirchner in US Patent 5206506, 1993.

15. March, R. E.; Hughes, R. J. Quadrupole Storage Mass Spectrometry; John Wiley and Sons: New York, 1989; p 24.

16. Schwartz, J. C.; Zhou, X.-G.; Bier, M. E. Method and Apparatus of Increasing Dynamic Range and Sensitivity of a Mass Spectrometer. US Patent 5572022, 1996.

17. Hager, J. W. Method of Reducing Space Charge in a Linear Ion Trap Mass Spectrometer. US Patent 6627876, 2003. 